\section{Desmoid abdominal tumor: A clinical case report and brief literature review}

\author{
Irina Ivanova, ${ }^{1}$ Shahswar Arif, ${ }^{2}$ \\ Dinko Dinev, ${ }^{1}$ Kalin Kalchev, ${ }^{3}$ \\ Maria Atanassova, ${ }^{1}$ Iskren Kotzev ${ }^{1}$ \\ 1 Clinic of Gastroenterology, Department \\ of Internal Diseases, St. Marina \\ Hospital, Varna; 2Medical University \\ "Dr. Paraskev Stoyanov", Varna; \\ ${ }^{3}$ Department of Pathology, St. Marina \\ Hospital, Varna, Bulgaria
}

\begin{abstract}
Desmoid tumors are unique mesenchymal neoplasm. They are able to spread to proximal tissues but tend not to metastasize. Our case presents a 66-year-old female referred for evaluation of the prominent, palpable mass located into the left abdomen. Imaging studies revealed a tumor up to 22 $\mathrm{cm}$, extending below the diaphragm to the retroperitoneal and intra-abdominal cavity. Contrast enhanced ultrasound showed strong inhomogeneous arterial hyper-enhancement followed by persistent enhancement in a venous phase. Histology obtained with trucut needle biopsy established desmoid tumor, with overall proliferating activity (Ki-67 expression) of $20 \%$. The lesion had been identified as sporadic and unresectable. During the patient's follow-up a slow but continuous elevation of serum creatinine was registered eventually led to anuria, requiring emergent hemodialysis. The non-obstructing nephropathy is an unusual complication of the disease course, therefore we briefly reviewed the published data on abdominal desmoid tumors and critically analyzed the relation with kidney injury.
\end{abstract}

\section{Introduction}

Desmoid tumors (DT) are rare neoplasms generating from myofibroblasts, categorized as deep aggressive fibromatoses. They include less than $4 \%$ of soft tissue injuries and less than $0.04 \%$ of all neoplasms. The biological characteristics of DT is complex and variable. They possess some features of malignant neoplasm like the ability to infiltrate the surrounding tissues and to reoccur after surgical removal, except the risk for metastatic growth. Furthermore, several studies reported a long-term stable disease and even spontaneous regression in some DT. ${ }^{1}$ In unresectable intra-abdominal DT the progres- sion of disease and local complications can be fatal. $^{2}$

\section{Case Report}

A 66-year-old Bulgarian female was referred to Clinic of Gastroenterology for evaluation of mild abdominal pain, fatigue and dyspnea during physical activity, loss of appetite, transitory nausea and vomiting and significant weight loss. The reported duration of symptoms was 3 weeks. She had medical history for ischemic heart disease, degenerative aortic valve stenosis, arterial hypertension (stage III, severe grade), bronchial asthma, osteoarthritis and type 2 diabetes (for approximately 10 years but under control with no registered complications related to disease). Family history for oncological diseases was negative. Physical exam revealed moderately impaired general condition, slight pallor of the skin, prominent, non-tender, hard in palpation mass started from the left hypochondria and extended bellow the umbilical line.

Abnormal laboratory tests registered: elevated ESR ( $45 \mathrm{~mm} / \mathrm{h}), \mathrm{CRP}(61 \mathrm{mg} / \mathrm{L})$, serum glucose level (ranged from 6.8 to 8.3 $\mathrm{mmol} / \mathrm{L}$ ), urea (up to $19.1 \mathrm{mmol} / \mathrm{L}$ ) and creatinine (up to $287 \mathrm{mcmol} / \mathrm{L}$ ), amylase (120 U/L, ULN $102 \mathrm{U} / \mathrm{L}$ ); urine was positive for proteins (+) with normal sediment. Hemoglobin level was $125 \mathrm{~g} / \mathrm{L}$; no change was observed in liver function tests, electrolytes, creatinine phosphokinase level and tumor markers (CEA, CA19-9, AFP).

Abdominal ultrasound revealed a heterogeneous, large, lobulated tumor formation of the left abdomen, spreading into retroperitoneum and peritoneal cavity, larger than 18 $\mathrm{cm}$ (Figure 1).

Contrast enhanced ultrasound (CEUS) after bolus injection of $2.4 \mathrm{~mL}$ SonoVue ruled out liver and spleen focal lesions. Furthermore, in arterial phase the large, irregular part of mass had become hyperechoic (gradual wash-in effect with a maximal enhancement at the end of arterial phase), whereas in portal phase, echogenicity was slightly less; contrast was more homogeneous, and lesion retained the contrast although in less grade for around 4 to $5 \mathrm{~min}$ utes of investigation. Thus, the typical washout phenomenon for malignant lesions was not observed.

Upper endoscopy excluded polypoid or tumor lesions of upper GIT. Multi-detector (MPR $3 \mathrm{~mm}$ ) computed tomography of the abdomen was performed only with oral contrast because of persistent elevation of creatinine. The tumor mass started below the left diaphragm, extending below and in front of the spleen; had relatively well-defined poly-
"St.Marina", 1 Hristo Smirnenski Blvd., Varna 9010, Bulgaria

Tel.: +359.52.978348 - Fax: +359.52.302891.

E-mail: irina.ivanova@mu-varna.bg

Key words: desmoid tumor, abdominal neoplasm; contrast enhanced ultrasound; nephropathy

Contributions: II and SA participated in the manuscript writing and preparation; all authors contribute to patient's evaluation and also as reviewers of the final content.

Conflicts of interest: the authors declare no potential conflict of interest.

Received for publication: 26 June 2017.

Accepted for publication: 28 September 2017.

This work is licensed under a Creative Commons Attribution NonCommercial 4.0 License (CC BY-NC 4.0).

(C) Copyright I. Ivanova, et al., 2017

Licensee PAGEPress, Italy

Gastroenterology Insights 2017; 8:6665

doi:10.4081/gi.2017.6665

cyclic borders, heterogeneous structure (noncontrast HU ranged 5 to 35) and enormous size - cranio-caudal diameter $22 \mathrm{~cm}$ (Figure $2)$. The lesion propagated into the retroperitoneum, was situated in front and below the duodenum, compressing the mesenteric vessels and left kidney, the later was caudally dislocated. The size of both kidneys was slightly and parenchyma of the left kidney showed atrophic changes with reduction to 5 $\mathrm{mm}$. There is no bowel loop dilations and thickening of the bowel wall, no significant lymph node enlargement and distant metastases.

At first, fine-needle aspiration biopsy of the mass was done, showing probable malignancy. The tru-cut needle biopsy of the tumor with ultrasound guidance was then performed. Histology reported evidence for proliferation of monomorphic elongated cells forming crossing fascicles, with moderate partially vacuolated cytoplasm, fusiform nuclei with prominent nucleoli, non-severe nuclear atypia; the stroma is consisting of network of vessel splits without erythrocytes (Figure 3). Immunohistochemistry investigation showed: negative stains for cytokeratin AE1/3, CD 117 and S-100; CD 34 was expressed only in vessel-like fissures; positive stains were D2-40, SMA and b-catenin there was mild to moderate globular cytoplasmic expression in tumor cells. Overall proliferation activity of the tumor, assessed with Ki-67 expression was $20 \%$, suggesting partial malignancy. Final histology report defines 
desmoid tumor - intra-abdominal deep fibromatosis.

Clinical differential diagnosis in our case includes: retroperitoneal fibrosarcoma; neuroendocrine tumor; gastrointestinal stromal tumor (GIST); neurofibroma and schwannoma.

The lesion had been identified as unresectable. The patient follow-up registered rapidly progressing fatigue, episodes of hypoglycaemia, decrease of diuresis to state of anuria, consequent rapid raise of creatinine to indication and start of emergent hemodialysis. Unfortunately, oncology committee decision was for palliative therapy, regarding the poor general condition and severe renal failure.

The patient provided written informed consent for planned investigations and use of the results at scientific observations.

\section{Discussion}

Desmoid tumors (DT) are also known as aggressive fibromatosis. Their development is a result of abnormal monoclonal proliferation of myofibroblasts. Usually they have benign microscopic appearance but intermediate biological behavior between fibrous lesions and sarcomas. DT are slowly growing and able to infiltrate surrounding tissues. There is a risk of recurrence after resection but no metastatic potential. DT typically present as large mass extending beyond the pseudo-capsule that they generate in. According to location they can be classified as intraabdominal, as part of abdominal wall or elsewhere (extra-abdominal). ${ }^{3}$ Intra-abdominal desmoids tend to invade mesenteric root and/or celiac axis and other abdominal organs. They are have a worse prognosis due to risk of complications as perforation, bleeding and bowel or ureteral obstruction. ${ }^{4}$

DT are very rare neoplasm, account for $0.03 \%$ of malignant diseases and have an incidence rate of 2 to 5 cases per million in European countries. 5,6 The associations with female gender and reproductive age, a history of abdominal surgery or trauma and family history of fibromatoses are well described. Disease more frequently occurs between puberty and fourth decade of life. Pregnancy can provoke development of DT, almost exclusively located in the abdominal wall. DT can be either sporadic or associated with inherited syndromes such as familial adenomatous polyposis (FAP), attenuated FAP or Gardner's syndrome. $10-25 \%$ of patients with FAP develop DT and these neoplasms are important cause for mortality after total colectomy. ${ }^{7}$ Most sporadic DT (85\%) have specific point mutations in exon 3 of gene coding beta-catenin (cadherin-associated pro-
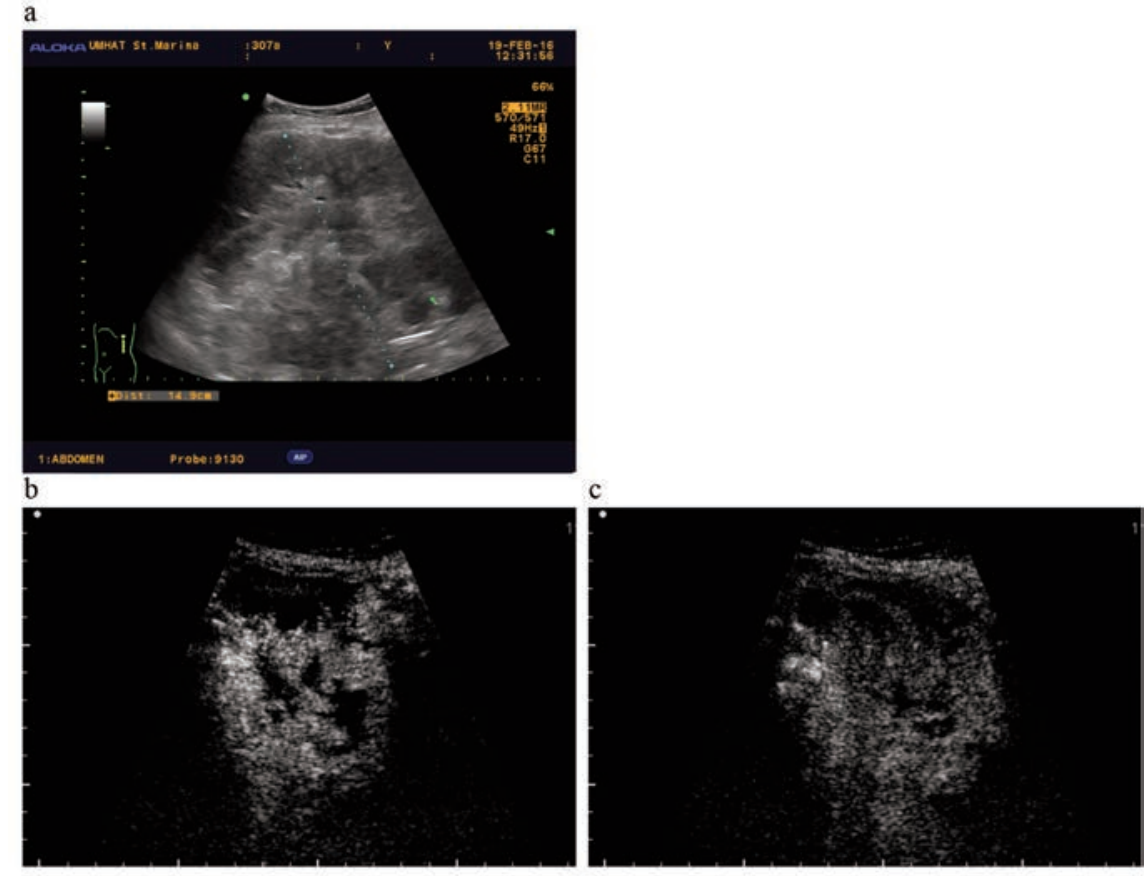

Figure 1. A) Ultrasound longitudinal scan at para-umbilical line showing heterogeneous tumor mass; B and C) Contrast enhanced ultrasound images of the tumor after $35 \mathrm{sec}$ and $75 \mathrm{sec}$ of SonoVue bolus.
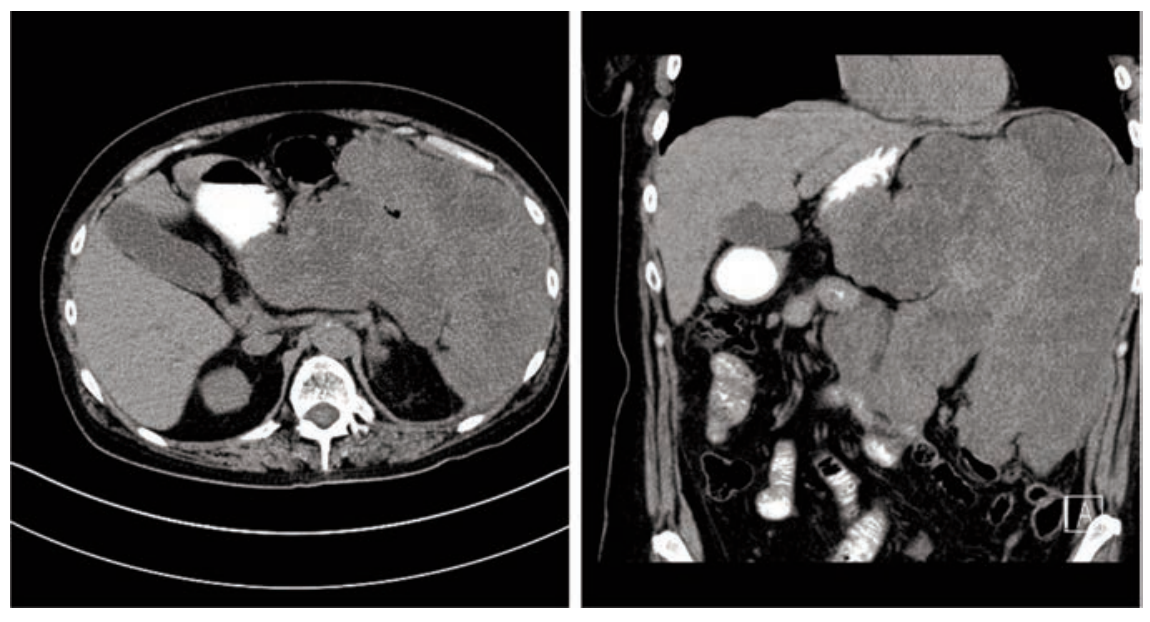

Figure 2. Transverse and coronal MD computed tomography abdominal scan.
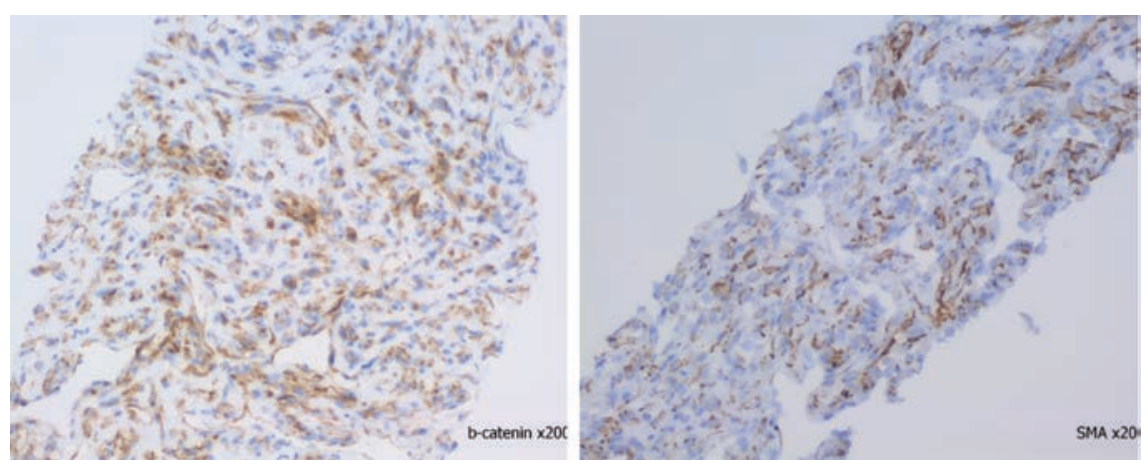

Figure 3. Histology findings, consistent with desmoid tumor. 
tein beta-1, CTNNB1), ${ }^{8}$ therefore betacatenin signaling may have an impact on DT growth and recurrence. ${ }^{9,10}$

Clinical presentations of abdominal DT includes local pain, palpable mass or local complications, like obstruction of the bowels, intestinal ischemia, fistulization, urethral compression with subsequent obstructive nephropathy. ${ }^{4}$ Magnetic resonance and $18 \mathrm{~F}$ fluorodeoxyglucose PET/CT scan help to characterize DT, but final diagnosis is based on histopathologic report. The mass is consisted of spindle cells, separated by collagen fibers. DT typically express nuclear betacatenin and show no expression of CD34 and CD117. Differential diagnosis with gastrointestinal stromal tumors, well-differentiated neuroendocrine tumors and low-grade sarcoma are very important.

Because of rarity of DT there are no clear management guidelines. The recommended approach is a multidisciplinary and the patients should be referred to a specialist sarcoma unit. ${ }^{11,12}$ Overall, radical (R0) surgery is the main option for treatment of intraabdominal DT and achieves a long-term disease-free period. Complete resection for advanced DT can be supported by plastic of abdominal wall by synthetic implant, vascular bypasses or even multivisceral transplantation. ${ }^{13,14}$ The recurrence of DT after surgical removal is important: rate varies from 19 to $57 \%$; median disease free survival is between 14 and 24 months. ${ }^{15}$ The factors found to be predictive of recurrence are: size $>4-5 \mathrm{~cm}$; age of the patient ( $<32-37$ years); microscopic negative margins after resection'16; CTNNB1 gene mutation S45F.9,10 Other therapeutic options, such as radiotherapy, hormonal therapy (tamoxifen, toremifene, anastrozole), biologic or targeted therapy (imatinib, low-dose interferon alpha), nonsteroidal anti-inflammatory drug intake (sulindac, celecoxib) or systemic chemotherapy (methotrexate and vincristine or doxorubicin-based regimens) can also be considered. ${ }^{1}$ Sorafenib has shown to be active in patients with progressive disease on chemotherapy. ${ }^{17}$ Local ablation techniques such as radiofrequency ablation, cryotherapy and HI-FU have also been studied with good results. Simple observation (wait-and-see policy) is another possible option for asymptomatic patients, because spontaneous stabilization or even regression may be achieved in some cases with extra-abdominal DT. ${ }^{12}$

We presented a 66-year-old female with a large, sporadic intra-abdominal DT, symptomatic at diagnosis, with a rapid deterioration of disease complicated with progressing renal failure. Looking up for causes of acute kidney injury the following conditions were excluded: obstruction of ureters; extra-renal dysfunction related to hypovolemia; use of nephrotoxic drugs; tumor lysis syndrome (spontaneous tumor cell necrosis), due to low proliferation activity of described lesion. In general, compression of the tumor may cause surrounding muscle tissue damage and crushlinked renal failure, however in our case serum Creatine kinase concentration, as a marker for that injury was normal. Therefore, unknown association of nephropathy and observed neoplastic disease can be suggested. Paraneoplastic syndrome can account for proteinuria and glomerular disease, a paraneoplastic glomerulopathy, but has been never described to be associated with DT. In general, glomerular injury is caused by secretion of tumor cell products, such as growth factors, cytokines, tumor antigens or hormones or by altered immune response in the presence of malignancy. ${ }^{18}$ Deterioration of patient's performance status and irreversible renal failure defined poor disease prognosis and failure to provide any curative treatment strategy.

\section{Conclusions}

To the best of our knowledge this is a first published report on clinical presentation of desmoid tumor in a Bulgarian patient. We can speculate on the role of paraneoplastic glomerulopathy for rapid deterioration of kidney function. Although extremely rare entity, DT should be included in the differential diagnosis of the abdominal neoplasms.

\section{References}

1. Mehren M von, Benjamin RS, Bui MM, et al. Soft tissue sarcoma, version 2.2012: featured updates to the NCCN guidelines. J Natl Compr Canc Netw 2012;10:95160.

2. Posner MC, Shiu MH, Newsome JL, et al. The desmoid tumor. Not a benign disease. Arch.Surg 1989;124:191-6.

3. Fletcher CDM, Bridge JA, Hogendoorn P, Mertens F. WHO Classification of Tumours, Volume 5. In: WHO Classification of tumors of soft tissue and bone.; 2013.

4. Rampone B, Pedrazzani C, Marrelli D, et al. Updates on abdominal desmoid tumors. World J Gastroenterol 2007;13:5985-8.

5. Penel N, Coindre JM, Bonvalot S, et al. Management of desmoid tumours: A nationwide survey of labelled reference centre networks in France. Eur J Cancer 2016;58:90-6.

6. Broekhoven DLM van, Grünhagen DJ, den Bakker MA, et al. Time trends in the incidence and treatment of extra-abdominal and abdominal aggressive fibromato- sis: a population-based study. Ann Surg Oncol 2015;22:2817-23.

7. Quintini C, Ward G, Shatnawei A, et al. Mortality of intra-abdominal desmoid tumors in patients with familial adenomatous polyposis: a single center review of 154 patients. Ann Surg 2012;255:5116.

8. Alman BA, Li C, Pajerski ME, et al. Increased beta-catenin protein and somatic APC mutations in sporadic aggressive fibromatoses (desmoid tumors). Am J Pathol 1997;151:329-34.

9. Colombo C, Miceli R, Lazar AJ, et al. CTNNB1 $45 \mathrm{~F}$ mutation is a molecular prognosticator of increased postoperative primary desmoid tumor recurrence: An independent, multicenter validation study. Cancer 2013;119:3696-702.

10. Lazar AJF, Tuvin D, Hajibashi S, et al. Specific mutations in the beta-catenin gene (CTNNB1) correlate with local recurrence in sporadic desmoid tumors. Am J Pathol 2008;173:1518-27.

11. ESMO/European Sarcoma Network Working Group. Soft tissue and visceral sarcomas: ESMO clinical practice guidelines for diagnosis, treatment and followup. Ann Oncol 2012;23;vii92-9.

12. Kasper B, Baumgarten C, Bonvalot S, et al. Management of sporadic desmoidtype fibromatosis: a European consensus approach based on patients' and professionals' expertise - a sarcoma patients EuroNet and European Organisation for Research and Treatment of Cancer/Soft Tissue and Bone Sarcoma Group initiative. Eur J Cancer 2015;51:127-36.

13. Khomiakov VM, Cheremisov VV, Chaika AV, et al. [Experience of surgical treatment of abdominal and intraabdominal desmoid fibromas]. Khirurgiia (Sofiia). 2014:17-25. [Article in Bulgarian]

14. Sakorafas GH, Nissotakis C, Peros G. Abdominal desmoid tumors. Surg Oncol 2007;16:131-42.

15. Lev D, Kotilingam D, Wei C, et al. Optimizing treatment of desmoid tumors. J Clin Oncol 2007;25:1785-91.

16. Sorensen A, Keller J, Nielsen OS, et al. Treatment of aggressive fibromatosis: a retrospective study of 72 patients followed for 1-27 years. Acta Orthop Scand 2002;73:213-9.

17. Gounder MM, Lefkowitz RA, Keohan ML, et al. Activity of Sorafenib against desmoid tumor/deep fibromatosis. Clin Cancer Res 2011;17:4082-90.

18. Pani A, Porta C, Cosmai L, et al. Glomerular diseases and cancer: evaluation of underlying malignancy. J Nephrol 2015;29:143-52. 